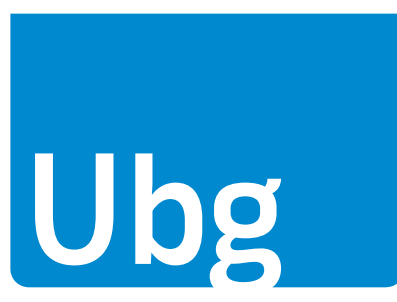

\title{
Die Unternehmensbesteuerung
}

\author{
Herausgeber \\ WP StB Prof. Dr. Thomas Rödder · RA Prof. Dr. Florian Haase, M.I.Tax
}

in Verbindung mit VRiBFH Prof. Dr. Roland Wacker · VRiBFH Michael Wendt · MDir. Dr. Rolf Möhlenbrock · MDig a.D. Dr. Steffen Neumann · Prof. Dr. Rainer Hüttemann · Prof. Dr. Christoph Spengel · Prof. Dr. Christian Dorenkamp, Deutsche Telekom · Mathias Gerner, Dr. Oetker KG.

\section{Beiratsmitglieder}

Dr. Martina Baumgärtel, Allianz SE · Peter Carstens, Otto GmbH \& Co. KG · WP, StB Hans-Martin Eckstein, PricewaterhouseCoopers AG · Christoph Felder, Ford-Werke GmbH · Brigitte Fischer, Freudenberg \& Co. KG · Prof. Dr. Guido Förster, Universität Düsseldorf · MDig. Volker Freund, Bayerisches Staatsministerium der Finanzen · RA, WP StB Prof. Dr. Wilhelm Haarmann, McDermott Will \& Emery · Dr. Wolfgang Haas, BASF SE · RA, StB Prof. Dr. Michael Hendricks, Flick Gocke Schaumburg · Dr. Berend Holst, Volkswagen AG · WP, StB Ralf Klaßmann, BDO AG · StB Prof. Dr. Stefan Köhler, Ernst \& Young GmbH · StB Dr. Michael Kröner, Universal Units GmbH · Prof. Dr. Robert Risse, Henkel AG \& Co. KGaA · Volker Schmidt-Fehrenbacher, Vodafone GmbH · StB Markus Suchanek, Flick Gocke Schaumburg · Werner Thumbs, Boehringer Ingelheim · RAin, StBin Dr. Heike Weber, Allen \& Overy · Dr. Monika Wünnemann, BDI e.V.

\section{Inhalt}

otto-schmidt.de/ubg

\section{Editorial}

Editorial - Neues Angebot eines Double blind Review Verfahrens für Aufsätze aus der Betriebswirtschaftlichen Steuerlehre ab dem Jahr 2021 ......

\section{Beiträge}

Prof. Dr. Florian Haase / Philip Nürnberg - Der Referentenentwurf eines Gesetzes zur Modernisierung der Entlastung von Abzugsteuern und der Bescheinigung von Kapitalertragsteuer

Mit dem sperrigen Titel "Gesetz zur Modernisierung der Entlastung von Abzugsteuern und der Bescheinigung von Kapitalertragsteuer" wäre das ohnehin Corona-bedrohte Weihnachtsfest 2020 gesichert. Die Kurzform Abzugsteuerentlastungsmodernisierungsgesetz (AbzStEntModG) macht es allerdings kaum besser. Auch inhaltlich hat das BMF kurz vor Jahresende und trotz vieler anderer laufender steuerlicher Gesetzgebungsverfahren, deren Ende man sich vorrangig herbeisehnt, eine schwerfällige Lektüre geschaffen. Der vorliegende Beitrag wirft einen ersten Blick auf die anstehenden Gesetzesänderungen.

\section{Dr. Ulf Andresen - Skandal im Sperrwirkungsbezirk 㯊}

Das fast gleichnamige Lied der Spider Murphy Gang setzt sich kritisch mit der rechtlichen Durchsetzung der Sperrbezirksverordnung der Stadt München auseinander, mit der in den Achtziger Jahren Prostitution aus deren Innenstadtbereich verbannt werden sollte. Die aktuelle Rechtsprechung des I. BFH-Senats zu Outbound-Konzernfinanzierungen hat das Potential, Unternehmen aus Deutschland im internationalen Wettbewerb in der Zukunft dauerhaft zu benachteiligen, weil sie unternehmerische Betätigungen wegen dieser Rechtsprechung nicht mehr so finanzieren können, wie es aus wirtschaftlicher und insbesondere (insolvenz)rechtlicher Sicht im Fremdvergleich geboten ist. Dem liegt, wie dieser Beitrag zeigt, ein eklatant falsches Wirtschafts- und Rechtsverständnis bei der Anwendung des Fremdvergleichs zugrunde, das zur Vertreibung inländischer Unternehmensgruppen in Länder außerhalb des vom I. BFH-Senat errichteten steuerwettbewerblichen "Sperrbezirks" führt. 
Inhalt

Dr. Lars H. Haverkamp, LL.M. (Christchurch)/Sara Meinert, LL.M. - Die Funktionsverlagerung dem Grunde nach

Der Beitrag nimmt die Entscheidung des FG München vom 26.11.2019, die sich mit streitigen Fragen zum Thema der Funktionsverlagerung auseinandersetzt, zum Anlass, auf die in der Praxis typischerweise auftretenden Fragestellungen im Zusammenhang mit der Prüfung einer Funk-

tionsverlagerung einzugehen.

\section{LRD Franz Hruschka - Ein Plädoyer für den Gewinn der personallosen Betriebsstätte}

Seit Einführung des AOA mit Wirkung ab 1.1.2013 stellt die Personalfunktion den zentralen Ankerpunkt für die Zuordnung von Gewinnen zu einer Betriebsstätte dar. Nach wie vor eröffnet § 1 Abs. 5 Satz 3 AStG die Möglichkeit, von diesem Prinzip in bestimmten Fällen abzuweichen. Solche sind nach Ansicht des Autors insbesondere dann gegeben, wenn entweder in einer personallosen Betriebsstätte die Hauptfunktion des Unternehmens betrieben wird oder im Gesamtunternehmen keine bedeutenden Personalfunktionen verrichtet werden.

\section{Stephan Kristl - Fahrzeugdaten - Erfassung im Konzern- und Einzelabschluss}

Fahrzeugdaten gelten (neben dem Algorithmus) als der zentrale Werttreiber datenbasierter Geschäftsmodelle in der Automobilindustrie, und damit der Zukunft. Vor diesem Hintergrund untersucht der Beitrag, ob durch Sensoren geschaffene, im Auto erhobene Daten im Konzernabschluss und/ oder Einzelabschluss zu bilanzieren sind. Wenn ja, als eigenständige Posten oder als Teil der Herstellungskosten einer urheberrechtlich geschützten Datenbank.

Manuel Brühl / Dr. Martin Weiss - Zur finanziellen Eingliederung bei der körperschaftsteuerlichen Organschaft nach einem Anteilstausch ( 21 UmwStG): FG Düsseldorf widerspricht der Auffassung der Finanzverwaltung

Immer wieder ist die Praxis bei Umwandlungen vor die Aufgabe gestellt, eine Kapitalgesellschaft durch die Umwandlung zu gründen, mit Betriebsvermögen auszustatten oder die Anteile an ihr umzuhängen und diese Kapitalgesellschaft unmittelbar organschaftlich an einen Organträger anzubinden. Als Hürde erweist sich hierbei beim Anteilstausch gem. § 21 UmwStG die Textziffer Org.15 des Umwandlungssteuererlasses, wonach die Organschaft im Verhältnis von übernehmender und erworbener Gesellschaft frühestens ab dem Beginn des auf die Einbringung folgenden Wirtschaftsjahrs begründet werden könne. Dass diese Beschränkung der Organschaft vom Gesetz nicht gedeckt ist, bestätigt nun das FG Düsseldorf in einer aktuellen Entscheidung.

\section{Rechtsentwicklung}

\section{Zeitschrift und Online-Datenbank gehören zusammen!}

Nutzen Sie die Online-Vorteile: Ihre neuen Zugangsdaten für 3 Nutzer finden Sie im Oktoberheft links neben dem Inhaltsverzeichnis. Auch für die Otto Schmidt Zeitschriften-App! Wichtige Info für bereits registrierte Nutzer: Verlängern Sie jetzt Ihren Zugriff mit den neuen Zugangsdaten!

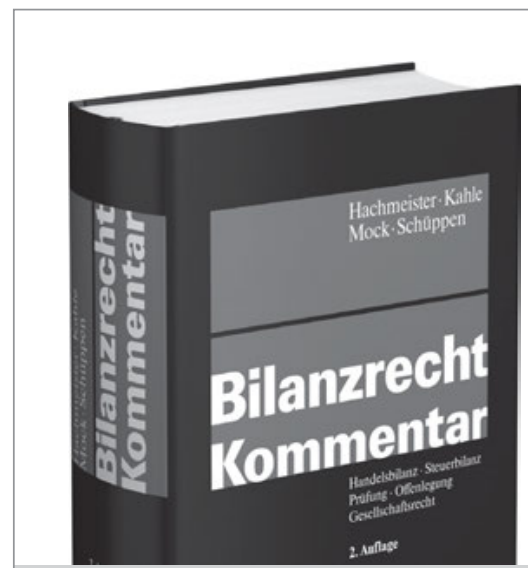

\section{Neuauflage}

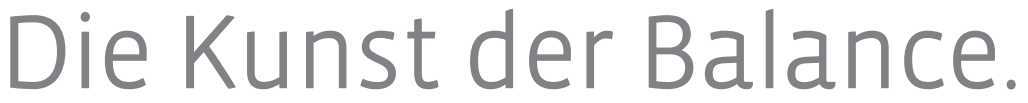

Mit diesem hochkarätigen Kommentar haben Sie den Jahresabschluss unter Kontrolle. Mit ARUG II, DCGK 2020 und COVID-19-Gesetzgebung. Besonderer Mehrwert: Handelsbilanzrecht, Steuerbilanzrecht und Gesellschaftsrecht in einem Werk. 